\title{
How Advances in Imaging Will Affect Precision Radiation Oncology
}

Citation for published version (APA):

Jaffray, D. A., Das, S., Jacobs, P. M., Jeraj, R., \& Lambin, P. (2018). How Advances in Imaging Will Affect Precision Radiation Oncology. International Journal of Radiation Oncology Biology Physics, 101(2), 292298. https://doi.org/10.1016/j.jijobp.2018.01.047

Document status and date:

Published: 01/06/2018

DOI:

10.1016/j.jijrobp.2018.01.047

Document Version:

Publisher's PDF, also known as Version of record

Document license:

Taverne

Please check the document version of this publication:

- A submitted manuscript is the version of the article upon submission and before peer-review. There can be important differences between the submitted version and the official published version of record.

People interested in the research are advised to contact the author for the final version of the publication, or visit the DOI to the publisher's website.

- The final author version and the galley proof are versions of the publication after peer review.

- The final published version features the final layout of the paper including the volume, issue and page numbers.

Link to publication

\footnotetext{
General rights rights.

- You may freely distribute the URL identifying the publication in the public portal. please follow below link for the End User Agreement:

www.umlib.nl/taverne-license

Take down policy

If you believe that this document breaches copyright please contact us at:

repository@maastrichtuniversity.nl

providing details and we will investigate your claim.
}

Copyright and moral rights for the publications made accessible in the public portal are retained by the authors and/or other copyright owners and it is a condition of accessing publications that users recognise and abide by the legal requirements associated with these

- Users may download and print one copy of any publication from the public portal for the purpose of private study or research.

- You may not further distribute the material or use it for any profit-making activity or commercial gain

If the publication is distributed under the terms of Article $25 \mathrm{fa}$ of the Dutch Copyright Act, indicated by the "Taverne" license above, 


\title{
PRECISION MEDICINE-COMMENTARY
}

\section{How Advances in Imaging Will Affect Precision Radiation Oncology}

\author{
David A. Jaffray, PhD, ${ }^{*}$ Shiva Das, PhD, * Paula M. Jacobs, PhD, ${ }^{*} \dagger$ \\ Robert Jeraj, PhD,* and Philippe Lambin, MD, PhD
}

\author{
*Department of Medical Physics, Princess Margaret Cancer Centre, Toronto, Ontario, Canada; \\ ${ }^{\dagger}$ National Institutes of Health, National Cancer Institute, Bethesda, Maryland; and ${ }^{\ddagger}$ The D-Lab: \\ Decision Support for Precision Medicine, GROW -School for Oncology, Maastricht University Medical \\ Centre+, Maastricht, the Netherlands
}

Received Mar 17, 2017, and in revised form May 11, 2017. Accepted for publication Jan 12, 2018.

\section{Summary}

The development of new imaging methods, the advancement of quantitative and validated imaging signals, the adoption of highly automated pipelines for analysis, and the integration with the many other "panomic" cancer features are critical for our success.

\begin{abstract}
Radiation oncology is 1 of the most structured disciplines in medicine. It is of a highly technical nature with reliance on robotic systems to deliver intervention, engagement of diverse expertise, and early adoption of digital approaches to optimize and execute the application of this highly effective cancer treatment. As a localized intervention, the dependence on sensitive, specific, and accurate imaging to define the extent of disease, its heterogeneity, and adjacency to normal tissues directly affects the therapeutic ratio. Image-based in vivo temporal monitoring of the response to treatment enables adaptation and further affects the therapeutic ratio. Thus, more precise intervention will enable fractionation schedules that better interoperate with advances such as immunotherapy. In the data set-rich era that promises precision and personalized medicine, the radiation oncology field will integrate these new data into highly protocoled pathways of care that begin with multimodality prediction and enable patient-specific adaptation of therapy based on quantitative measures of the individual's dose-volume temporal trajectory and midtherapy predictions of response. In addition to advancements in computed tomography imaging, emerging technologies, such as ultra-high-field magnetic resonance and molecular imaging will bring new information to the design of treatments. Next-generation image guided radiation therapy systems will inject high specificity and sensitivity data and stimulate adaptive replanning. In addition, a myriad
\end{abstract}

Reprint requests to: David A. Jaffray, PhD, Department of Medical Physics, Princess Margaret Cancer Centre, 5-631, 610 University Ave, Toronto, ON M5G2M9, Canada. Tel: (416) 946-2387; E-mail: david. jaffray@ rmp.uhn.on.ca

Conflict of interest: Dr Jaffray reports grants/sponsored research agreements from Raysearch Laboratories, Philips Medical Systems, Elekta, Inc, Varian Medical Systems, Siemens Medical, and IMRIS; presenter fees from the American Society of Therapeutic Radiation Oncology; and royalties from Modus Medical and Precision X-ray related to nonpatentable inventions, outside the submitted work. Dr Jaffray has the following pending patents: US 2013/026137 A1, US61/178,319, US61/ 157,738, and US2013/0113802 A1; the following issued patients: 7399977, US11/867998, and PCT/US2007/067847; the following issued patents licensed to Elekta, Inc: 8,039,790 (with royalties received), 20040234115, 20040096038, 20040218719, 7472765 (with royalties received), and 7,147,373 (with royalties received); and the following issued patents licensed to iRT: US60/806842, PCT/CA2007/001209, and EP20070763872. Dr Lambin reports grants/sponsored reserach from Oncoradiomics. Dr Lambin is inventor of two patents on radiomics and has shares in the company Oncoradiomics. 
of pre- and peritherapeutic markers derived from advances in molecular pathology (eg, tumor genomics), automated and comprehensive imaging analytics (eg, radiomics, tumor microenvironment), and many other emerging biomarkers (eg, circulating tumor cell assays) will need to be integrated to maximize the benefit of radiation therapy for an individual patient. We present a perspective on the promise and challenges of fully exploiting imaging data in the pursuit of personalized radiation therapy, drawing from the presentations and broader discussions at the 2016 American Society of Therapeutic Radiation Oncology-National Cancer Institute workshop on Precision Medicine in Radiation Oncology (Bethesda, MD). (c) 2018 Published by Elsevier Inc.

\section{Introduction}

The advent of computed tomography (CT), magnetic resonance (MR) imaging, and positron emission tomography (PET) in the past 50 years has transformed the detection and treatment of cancer. These technologies have had a profound impact on our ability to better predict the outcomes of existing interventions and enabled a period of remarkable innovation, bringing new interventions and paradigms of care. The continued pace of advancement in imaging technology, combined with our growing ability to analyze the data from individual patients and aggregate these data across large patient cohorts, is creating a future in which care is becoming increasingly precise and personalized. Thus, the intervention is optimized according to welldefined patient-specific measurement regimens (eg, imaging, genomics, epigenomics) that have been derived from aggregated learning through large cohort data sets. The minimally invasive nature of imaging portrays its unique and critical role as an enabler of precision medicine in radiation oncology-whether that precision is in better defining the patient-specific extent of disease or being used in combination with many other inputs to design the timedependent pattern of dose to be applied.

Although imaging has always played a central role in radiation oncology - as evidenced by most radiation oncology departments starting as divisions within the radiology department-imaging and image-derived signals will be even more integral to future practice. Imagederived features (eg, tumor size, location, imaging phenotype, texture) are now affecting nearly every facet of patient care and are used at every step (eg, diagnosis, daily treatment delivery, response assessment) in the process. Figure 1 illustrates the many image-related activities in the processes of patient characterization, decision making, care delivery, and follow-up phases of a patient's journey through modern radiation therapy. Isolating the effect of advances in imaging is challenging, given the wide diversity of roles it plays. Furthermore, advances in 1 domain can enhance or diminish the importance of imaging in another. The development of MR-guided radiation therapy systems is an example in which consolidation of a diagnostic MR scanner with a linear accelerator might enable integrated delineation, adaptation, and response assessment from a single system (ie, daily MR imaging of the patient during treatment) (1). The schematic in Figure 1 also highlights 2 other important concepts-intent and information flow. With the emergence of rich new information during the course of therapy, the clinician must focus on clinical intent instead of "prescription." Although previously considered "tampering" with evidence-based practice, the idea of adaptation within the course of therapy becomes a potential reality with the emergence of robust, noninvasive imaging signals, including anatomic and functional changes $(2,3)$. The field of radiation oncology has been a "first mover" in this area, with the development of adaptive radiation therapy enabled by imageguidance systems $(4,5)$. The effective and efficient flow of information (Fig. 1) is central to enabling this paradigm. Robust data exchange standards, gigabit data transfer speeds, on-demand computing capacity, artificial intelligence-based automation methods, and deformation technologies are enablers, and their scale up in the era of "big data" will open the door for greater consumption of imaging data in the field of radiation oncology (6). The success of the precision medicine paradigm requires that these diverse imaging data be drawn together with genomic data (including epigenetics), phenotypic data (eg, tumor microenvironment), emerging models of radiation biology (eg, radiogenomics, immunotherapy), and, of utmost importance, clinical outcomes.

In June 2016, the American Society of Radiation Oncology, National Cancer Institute, and American Association of Physicists in Medicine co-sponsored a 2-day workshop for radiation oncology physicians and medical physicists focused on precision medicine in radiation oncology. The workshop provided leaders the opportunity to interact with their peers and identify a new foundation of conducting research to bring the precision medicine paradigm to radiation oncology. Specific insights were sought regarding the future scientific direction of radiation oncology, the digital infrastructure needed to participate, and the practical and logistical barriers to enabling precision medicine in radiation oncology. Presentations and dialogue concentrated on genomics, imaging, big data, and real-world challenges to advance precision medicine in radiation oncology research, quality assurance, and safety. In addition to scientific posters and oral presentations from experts in the field, a dedicated workshop session for each 


\section{Multiple Roles of Imaging in the Multiple Timescales of Precision Cancer Medicine}

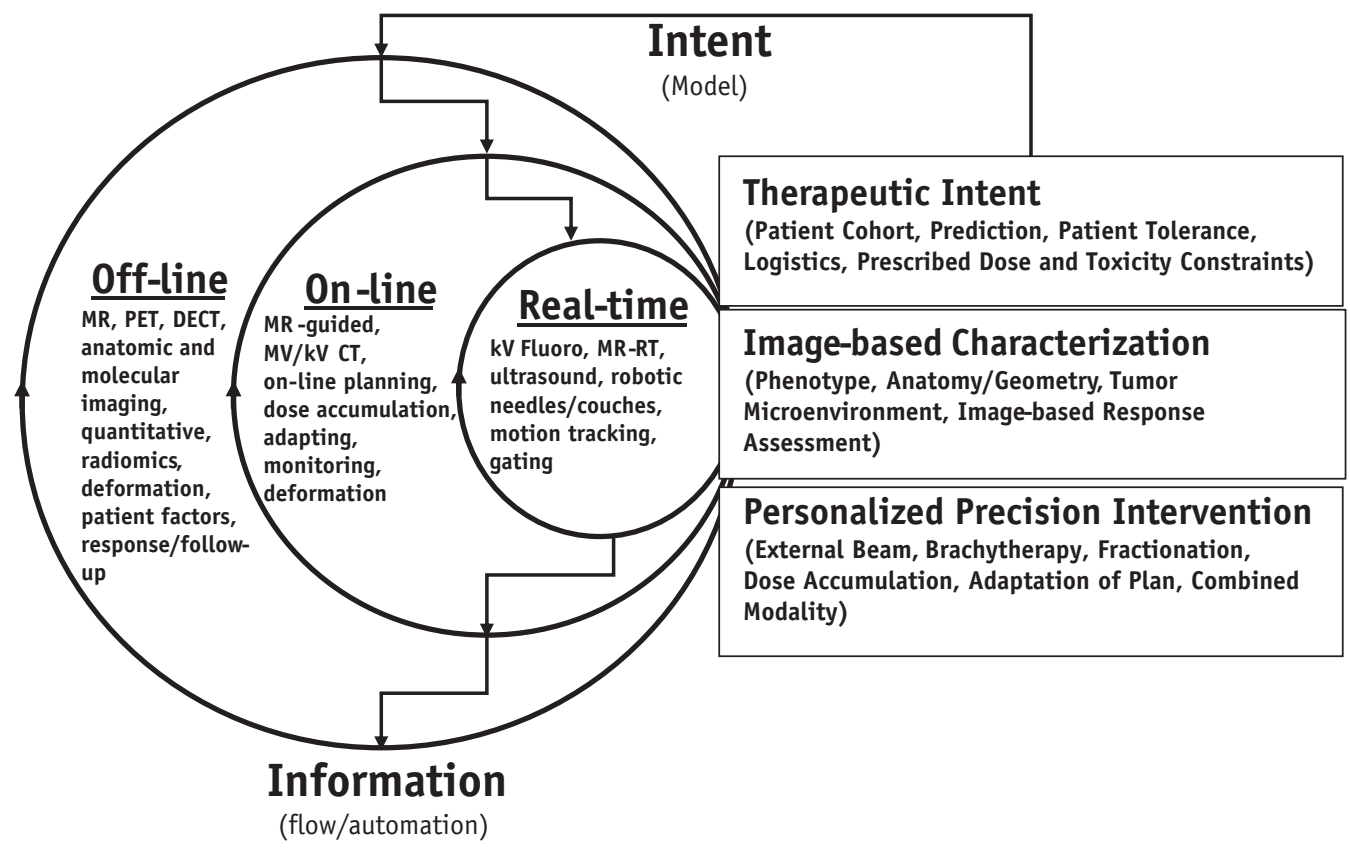

Fig. 1. Multiple roles of imaging in the multiple timescales of personalizing radiation therapy. The practice of radiation oncology uses imaging data at every point in the process, including characterization of the patient and his or her disease, prediction of the outcome, design of the dose distribution, targeting and adaptation, real-time tracking, and response assessment. Image-based information informs the intent and enables patient-specific personalization of the treatment. In the absence of precision, this process will not build evidence nor increase our understanding of the disease or the effectiveness of our interventions. Pursuing the precision medicine paradigm requires us to improve our models of response and automate the flow of information.

of the major themes identified opportunities, challenges, and future directions to advance the precision cancer medicine paradigm. The key imaging themes presented and workshop outcomes are presented in this report.

\section{Rapid Pace of Advances in Imaging-Measurement Is King}

Molecular imaging approaches have long held the promise of augmenting the anatomic approach currently used for the design of highly conformal radiation dose distributions (7). The science of radiobiology, augmented by the ability of molecular imaging to assess the spatial heterogeneity of tumors, provided the basis for this approach, with decades of evidence highlighting the variation in the radiosensitivity of cells depending on the type, location in the cell cycle, and microenvironment (8). Without the ability to localize phenotypic heterogeneity, the entire tumor must be dosed to some minimum dose to ensure the most resistant disease is controlled. The effect of this approach on the adjacent normal tissues and the subsequent determination of patient-specific tolerance to a course of radiation therapy severely limited the development of more personalized radiation therapy regimens.
For the purposes of our report, focusing on the topic of hypoxia will be illustrative. The effect of very low oxygen levels on cell radiosensitivity has been established in basic science and is a well-documented negative predictive factor in the outcomes of a variety of cancers, including cancer of the cervix, head and neck, and prostate (9-12). Although most of these studies were performed using an invasive oxygen sensing Eppendorf probe, others have used PET imaging approaches to determine the magnitude and distribution of hypoxia in the tumor (13). In addition to giving valuable input into patient cohort classification, these results also highlight the potential to design hypoxiaescalating and normoxia de-escalating dose distributions $(14,15)$. Implicit in this approach is the assumption that these molecular patterns are accurate representations of the underlying biology and are stable throughout the course of radiation therapy $(16,17)$ (Fig. 2).

These exciting advances in molecular imaging of hypoxia raise many questions and define key areas of research for the inclusion of molecular imaging into the field of radiation oncology. Do we have the spatial resolution in our application of dose that is consistent with the imaging findings, and does the imaging modality have the ability to spatially resolve the underlying biology? (18). Do we consider these signals as absolute quantities, or do 


\section{Pre-radiation therapy Mid-radiation therapy}

\section{Post-radiation therapy}

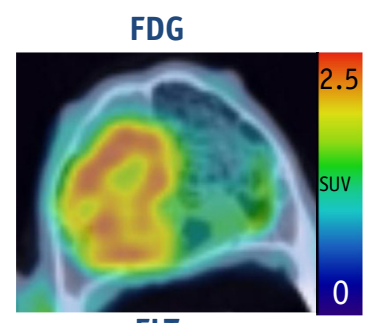

FLT

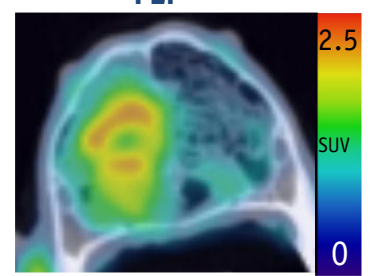

CU-ATSM

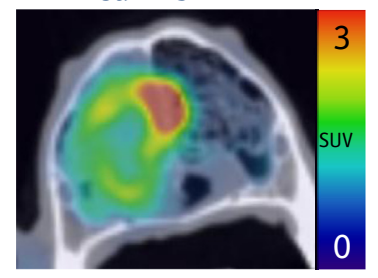

FLT

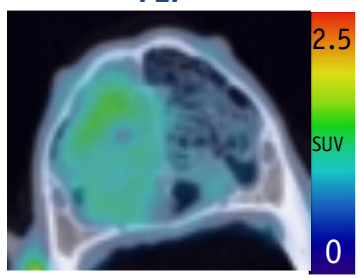

Cu-ATSM

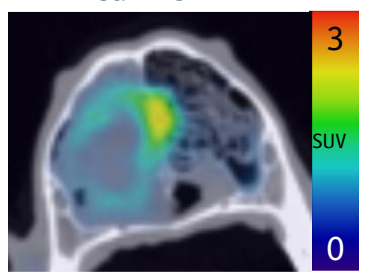

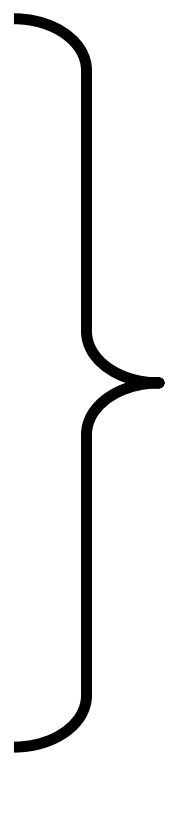

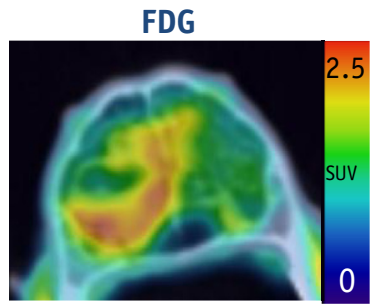

Fig. 2. Preclinical research in multimodality molecular imaging emphasizes the complex response of the disease before, during, and after radiation therapy (17). Using fludeoxyglucose positron emission tomography/computed tomography (PET/ $\mathrm{CT}$ ) as a surrogate of metabolism, fluorothymidine PET/CT as a surrogate of proliferation, and Cu-ATSM PET/CT as a surrogate of hypoxia scans at different time points in a canine nasosinus model demonstrates: (1) a high degree of spatial heterogeneity within tumors; (2) the complexity of the tumor response; and (3) our ability to monitor and potentially target the spatiotemporal heterogeneity of tumors to maximize the therapeutic ratio in radiation therapy. Imaging has the potential to both personalize treatments and inform the next generation of radiobiological models.

we consider time-dependent changes as more relevant in the fractionation paradigm of radiation therapy? (19). We are also becoming increasingly aware of the heterogeneity of the biology within tumors. Should we integrate other molecular or physiological signals-metabolism as measured through fludeoxyglucose PET or proliferation as measured through fluorothymidine PET - to pursue an even more accurate description of the underlying biology? (20). If we have an accurate description of the underlying biology, how do we derive the corresponding dose regimen and distribution? (21). Even the simple linkage between an image of hypoxia and the resulting dose distribution requires maturation. It is also important that the field consider the intrinsic uncertainties in the process of radiation therapy delivery when defining its objectives and design integrated solutions that are robust for clinical application.

The intrinsically localized nature of radiation therapy requires the field to be extremely attentive to sensitivity- and specificity-related advances in cancer imaging. More specifically, technologies that are directly "adjacent" to our current practice in terms of technology or care delivery can have immediate effects. Two exciting technologies that illustrate this point are highlighted in Figure 3 (22). First, the development of a highly specific form of prostate-specific membrane antigen that has the potential to affect the care of recurrent prostate cancer; and, second, the commercial development of $7 \mathrm{~T}$ MR imaging systems $(23,24)$. In the case of novel prostate-specific membrane antigen agents, the potential to monitor and target recurrent disease is as important as the potential to use these agents to better delineate focal disease in the prostate gland for localized treatment. The potential effect of 7T imaging of the brain for detection, decision to treat, and localization of primary and metastatic lesions in the context of stereotactic radiosurgery is likely but will require investment to characterize the geometric accuracy at these field strengths.

\section{Image Validation-No More Pretty Pictures}

The ingestion of new imaging signals into the radiation therapy process is a significant challenge. Although the field was aggressive in adopting CT imaging for definition, delineation, and targeting, we have experienced significantly slower uptake of other modalities, including MR and molecular imaging. This is arguably in part owing to the challenges the field has had in ensuring the geometric 


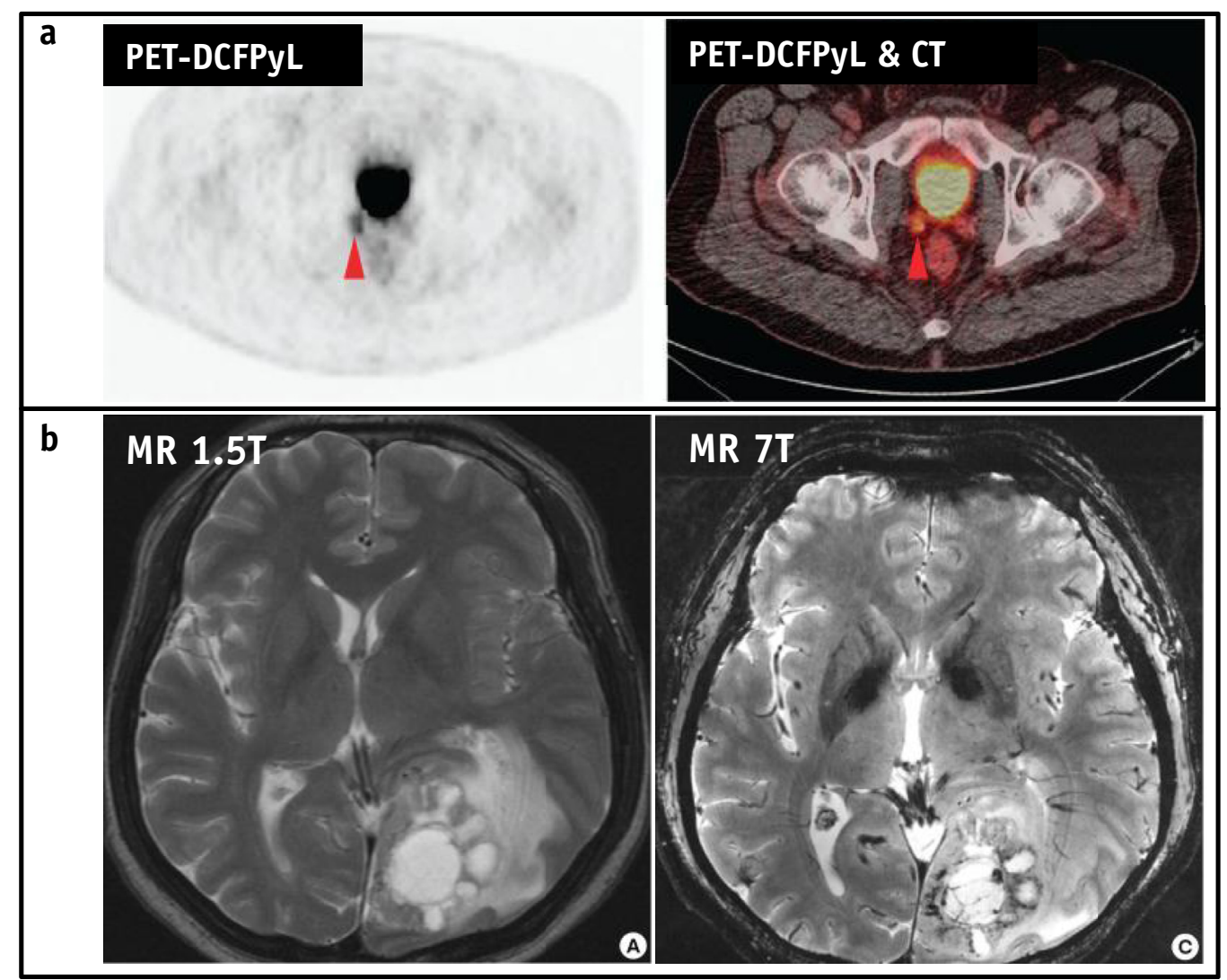

Fig. 3. (a) ${ }^{18} \mathrm{~F}$-DCFPyL positron emission tomography (PET) and PET/computed tomography (CT) images from a patient with biochemical recurrence after radical prostatectomy (prostate-specific antigen [PSA] $0.3 \mathrm{ng} / \mathrm{dL}$ at imaging). Subtle increased radiotracer uptake can be seen in the right prostate bed (red arrowheads) compatible with local disease recurrence (uptake in the urinary bladder is present anteromedial to the local recurrence). The patient subsequently underwent salvage radiation therapy with a boost to the region of ${ }^{18} \mathrm{~F}$-DCFPyL uptake, and the PSA level became undetectable. The lack of radiotracer uptake outside the typical salvage pelvic radiation field increased the confidence level of the treating clinicians that salvage radiation therapy was the appropriate course of action for this patient (22). (b) The effect of continued advances in imaging performance on radiation therapy will directly alter patient care. This is illustrated in the comparison of magnetic resonance images of a metastatic brain tumor of non-small cell lung carcinoma acquired at conventional (1.5T) and high-field strength (7T). T2-weighted axial image from 1.5T brain magnetic resonance image "(A)" and T2-weighted axial image from 7.0T brain magnetic resonance image "(C)" in a patient with a metastatic brain tumor of non-small cell lung carcinoma (23). (A color version of this figure is available at www.redjournal.org.)

and signal accuracy of these new signals and the expense and availability of these modalities. In many ways, CT was easy to adopt owing to its intrinsic geometric and radiometric precision (ie, Hounsfield units). MR imaging provides exquisite sensitivity but has lacked in specificity and geometric integrity, thereby delaying its broad uptake in the planning process. PET imaging, in contrast, provides exquisite sensitivity and specificity but lacks broad availability of radiopharmaceuticals beyond fludeoxyglucose, thereby generally limiting it to academic centers. The field needs to invest in a more formal image ingestion process if these new imaging signals are going to have the effect on patient care that is possible (25). Such a formalization would bring bright minds and industry partners to bear on the current clinically validated problems. It would also provide a "systems thinking" framework to balance the tradeoffs between greater accuracy in delineation against greater precision in dose delivery.

Specific challenges that have been identified include (1) the need for greater transparency in image-signal processing to ensure quantitative performance of the imaging system and quantitative consistency between imaging systems of the same modality used in a trial; (2) addressing the problems of image distortion and registration; and (3) pathology-based validation of the image-derived signals. The development of the NIH's Quantitative Imaging Network is an excellent example of the broader community's awareness of the limiting nature these challenges have on the field of cancer (26).

In addition to quantification, validation of the imaging signals must also be aggressively pursued. A founder of modern medical education and practice, Sir William Osler stated it 
clearly "as is our pathology, so goes our practice"-clearly, we must be cautious not to blindly "treat to the image." Although the number of reports on pathology-based validation is small compared with those promoting a novel imaging technique, an increase has occurred in the report of pathology-imaging correlation studies enabled through 3-dimensional mapping technologies $(26,27)$. The recent report by Menard et al (28) on the correlation between multiparametric MR and biopsies of the prostate gland highlights the scale of the problem, because fused multiparametric MR imaging was inadequate to delineate the tumor boundaries for focal salvage in 10 of 19 patients. Furthermore, the use of a 5-mm expansion margin only reduced this cohort to 7 of 19 patients. This is exciting work because it highlights the field's growing capabilities to quantify and validate image-based measurement of disease- a critical step toward image-enabled precision radiation oncology.

\section{Automation-Managing the 4 ' $\mathrm{V}$ 's of Imaging Data}

The volume, variety, velocity, and veracity of imaging data speak to the scale of the current and future challenges to integrating imaging data into precision radiation oncology. Managing the volume and variety requires alternative approaches to traditional expert observer-based analysis. Radiation oncology has been forced to be a leader in the field of automated delineation to respond to the power of inverse planning for intensity modulated radiation therapy. The burden of delineating normal tissues and target volumes has created a variety of algorithms and software tools that are now in routine use in the clinical setting (29). Similarly, automated methods are being successfully applied to extract and validate features that are beyond the quantification skills of the expert. This is highlighted in the birth of the field of "radiomics." Although the extraction of phenotype data from images is not novel, the extraction of features that are too subtle in their intensity or their frequency of occurrence for human experts to detect is now emerging as a predictive biomarker of response $(30,31)$. Although the clinical effect of this work is intriguing, the technical effect of this type of work needs to be recognized for its foreshadowing of cancer medicine and science. The process involved in radiomic analysis requires a massive scale-up in automation to manage the multitude of potentially relevant features, the need to integrate segmentation data, the number of image sets required to reach statistical significance, and the processes for regression analysis and machine learning. Further investments in automation will be required to allow these tools to contribute to clinical decision making, especially as time-dependent signatures become part of the equation. It is likely that robust clinical decision-making tools will need to integrate semantic, algorithm-derived, and machine learning-generated features with more traditional clinical information for maximum patient benefit. As illustrated in Figure 1, the volume, variety, and velocity of data flowing back from the intervention to the decision-making process will require automation. The veracity of the data refers to its quality and affects the certainty with which the data can be acted on-this is of critical importance given the life and death decisions being made in the design of a cancer patient's treatment.

The adoption of automated "data mining" approaches within the real-world setting of data-rich clinical care will challenge the conventional paradigm of hypothesis-based scientific research. Collecting and analyzing data for patterns and correlations without a predefined mechanistic or conceptual hypothesis has been enabled, and the field of clinical science and the processes of regulatory approval will need to wrestle with the incongruence with their traditional approaches. This was a specific topic of discussion at the Workshop, and it was recognized that a large amount of work must be done on both sides of the debate.

\section{Open Science-Breaking Down the Silos}

The American Society of Clinical Oncology proposed the term "panomics" to describe the seemingly endless list of measureable quantities of potential importance in the study of cancer and the delivery of modern cancer care. The broad scope of panomics speaks to not only the common interests but also the common methods that the scientific and clinical communities should be using to analyze the data sets found in clinical and basic science of oncology. The creation of a community of researchers that see their data as "features," regardless of their source-deep sequencing, 3-dimensional CT imaging data, dose-volume histograms, or clinical outcome data-is timely and powerful. Efforts by the National Institutes of Health to create powerful archives are ideal mechanisms to advance these efforts. The Cancer Imaging Archive and The Cancer Genomics Archive are linked with numerous site-specific research groups in bladder, breast, glioma, head and neck, ovarian, and renal cancers collaborating and reporting impactful research findings (32). Paradoxically, although it is work led by researchers in radiation oncology that are demonstrating the value of these "panomic" approaches $(33,34)$, a need exists for more radiation oncology data sets to be added to the archive. The field of radiation oncology needs to engage fully in the open science oncology community. Progress has been made in this regard through the medical physics archive on arXiv (available at: https://arxiv.org/list/physics.med-ph/ recent), where a variety of data sets are available for further analysis. Although exciting, the amount of data is a tiny fraction of the volume of well-curated imaging and dosimetry data sets that sit within individual academic radiation oncology programs. These need to be annotated and drawn together with the corresponding pools of genomic sequencing data in national archives to allow this research to be more fruitful and more broadly effective. 


\section{References}

1. Raaymakers BW, Lagendijk JJ, Overweg J, et al. Integrating a $1.5 \mathrm{~T}$ MRI scanner with a 6 MV accelerator: Proof of concept. Phys Med Biol 2009;54:N229-N237.

2. van Elmpt W, Ollers M, Dingemans AM, et al. Response assessment using 18F-FDG PET early in the course of radiotherapy correlates with survival in advanced-stage non-small cell lung cancer. J Nucl Med 2012;53:1514-1520.

3. van Kranen S, Hamming-Vrieze O, Wolf A, et al. Head and neck margin reduction with adaptive radiation therapy: Robustness of treatment plans against anatomy changes. Int $J$ Radiat Oncol Biol Phys 2016;96:653-660.

4. Schwartz DL, Garden AS, Thomas J, et al. Adaptive radiotherapy for head-and-neck cancer: Initial clinical outcomes from a prospective trial. Int J Radiat Oncol Biol Phys 2012;83:986-993.

5. Jaffray DA. Image-guided radiotherapy: From current concept to future perspectives. Nat Rev Clin Oncol 2012;9:688-699.

6. Benedict SH, El Naqa I, Klein EE, et al. Introduction to big data in radiation oncology: Exploring opportunities for research, quality assessment, and clinical care. Int J Radiat Oncol Biol Phys 2016;95:871-872.

7. Ling CC, Humm J, Larson S, et al. Towards multidimensional radiotherapy (MD-CRT): Biological imaging and biological conformality. Int J Radiat Oncol Biol Phys 2000;47:551-560.

8. Bradshaw TJ, Bowen SR, Jallow N, et al. Heterogeneity in intratumor correlations of ${ }^{18} \mathrm{~F}-\mathrm{FDG},{ }^{18} \mathrm{~F}-\mathrm{FLT}$, and ${ }^{61} \mathrm{Cu}$-ATSM PET in canine sinonasal tumors. J Nucl Med 2013;54:1931-1937.

9. Milosevic M, Warde P, Ménard C, et al. Tumor hypoxia predicts biochemical failure following radiotherapy for clinically localized prostate cancer. Clin Cancer Res 2012;18:2108-2114.

10. Nordsmark M, Bentzen SM, Rudat V, et al. Prognostic value of tumor oxygenation in 397 head and neck tumors after primary radiation therapy: An international multi-center study. Radiother Oncol 2005;77:18-24.

11. Fyles A, Milosevic M, Pintilie M, et al. Long-term performance of interstitial fluid pressure and hypoxia as prognostic factors in cervix cancer. Radiother Oncol 2006;80:132-137.

12. Lalonde E, Ishkanian AS, Sykes J, et al. Tumour genomic and microenvironmental heterogeneity for integrated prediction of 5-year biochemical recurrence of prostate cancer: A retrospective cohort study. Lancet Oncol 2014;15:1521-1532.

13. Mortensen LS, Johansen J, Kallehauge J, et al. FAZA PET/CT hypoxia imaging in patients with squamous cell carcinoma of the head and neck treated with radiotherapy: Results from the DAHANCA 24 trial. Radiother Oncol 2012;105:14-20.

14. Jeraj R, Bradshaw T, Simončič U, et al. Molecular imaging to plan radiotherapy and evaluate its efficacy. J Nucl Med 2015;56:1752-1765.

15. Rockne RC, Trister AD, Jacobs J, et al. A patient-specific computational model of hypoxia-modulated radiation resistance in glioblastoma using 18F-FMISO-PET. J R Soc Interface 2015;12. 20141174.

16. Lin Z, Mechalakos J, Nehmeh S, et al. The influence of changes in tumor hypoxia on dose-painting treatment plans based on 18F-FMISO positron emission tomography. Int J Radiat Oncol Biol Phys 2008;70: $1219-1228$.

17. Bradshaw TJ, Yip S, Jallow N, et al. Spatiotemporal stability of $\mathrm{Cu}-\mathrm{ATSM}$ and FLT positron emission tomography distributions during radiation therapy. Int J Radiat Oncol Biol Phys 2014;89: 399-405.

18. Bowen SR, van der Kogel AJ, Nordsmark M, et al. Characterization of positron emission tomography hypoxia tracer uptake and tissue oxygenation via electrochemical modeling. Nucl Med Biol 2011;38:771-780.

19. Gregoire V, Jeraj R, Lee JA, et al. Radiotherapy for head and neck tumours in 2012 and beyond: Conformal, tailored, and adaptive? Lancet Oncol 2012;13:e292-e300.

20. Nyflot MJ, Kruser TJ, Traynor AM, et al. Phase 1 trial of bevacizumab with concurrent chemoradiation therapy for squamous cell carcinoma of the head and neck with exploratory functional imaging of tumor hypoxia, proliferation, and perfusion. Int J Radiat Oncol Biol Phys 2015;91:942-951.

21. Bowen SR, Flynn RT, Bentzen SM, et al. On the sensitivity of IMRT dose optimization to the mathematical form of a biological imagingbased prescription function. Phys Med Biol 2009;54:1483-1501.

22. Rowe SP, Gorin MA, Allaf ME, et al. PET imaging of prostate-specific membrane antigen in prostate cancer: Current state of the art and future challenges. Prostate Cancer Prostatic Dis 2016;19:223-230.

23. Paek SL, Chung YS, Paek SH, et al. Early experience of pre- and postcontrast 7.0T MRI in brain tumors. J Korean Med Sci 2013;28:1362-1372.

24. Rowe SP, Macura KJ, Mena E, et al. PSMA-based [(18)F]DCFPyL $\mathrm{PET} / \mathrm{CT}$ is superior to conventional imaging for lesion detection in patients with metastatic prostate cancer. Mol Imaging Biol 2016;18: 411-419.

25. Jaffray DA, Chung C, Coolens C, et al. Quantitative imaging in radiation oncology: An emerging science and clinical service. Semin Radiat Oncol 2015;25:292-304.

26. Clarke LP, Nordstrom RJ, Zhang H, et al. The quantitative imaging network: NCI's historical perspective and planned goals. Transl Oncol 2014;7:1-4.

27. Gibson E, Bauman GS, Romagnoli C, et al. Toward prostate cancer contouring guidelines on magnetic resonance imaging: Dominant lesion gross and clinical target volume coverage via accurate histology fusion. Int J Radiat Oncol Biol Phys 2016;96:188-196.

28. Menard C, Iupati D, Publicover J, et al. MR-guided prostate biopsy for planning of focal salvage after radiation therapy. Radiology 2015;274: 181-191.

29. Sharp G, Fritscher KD, Pekar V, et al. Vision 20/20: Perspectives on automated image segmentation for radiotherapy. Med Phys 2014;41: 050902.

30. Diehn M, Nardini C, Wang DS, et al. Identification of noninvasive imaging surrogates for brain tumor gene-expression modules. Proc Natl Acad Sci U S A 2008;105:5213-5218.

31. Lambin P, Rios-Velazquez E, Leijenaar R, et al. Radiomics: Extracting more information from medical images using advanced feature analysis. Eur J Cancer 2012;48:441-446.

32. Kalpathy-Cramer J, Freymann JB, Kirby JS, et al. Quantitative imaging network: Data sharing and competitive algorithm validation leveraging The Cancer Imaging Archive. Transl Oncol 2014;7:147-152.

33. Aerts HJ, Velazquez ER, Leijenaar RT, et al. Decoding tumour phenotype by noninvasive imaging using a quantitative radiomics approach. Nat Commun 2014;5:4006.

34. Lambin P, van Stiphout RG, Starmans MH, et al. Predicting outcomes in radiation oncology-multifactorial decision support systems. Nat Rev Clin Oncol 2013;10:27-40. 